\title{
Politique
}

\section{De la tour d'ivoire à la place publique}

\section{Gérard Bergeron}

Volume 1, numéro 1, janvier 1982

Les intellectuels et les pouvoirs

URI : https://id.erudit.org/iderudit/040389ar

DOI : https://doi.org/10.7202/040389ar

Aller au sommaire du numéro

Éditeur(s)

Société québécoise de science politique

ISSN

0711-608X (numérique)

Découvrir la revue

Citer cet article

Bergeron, G. (1982). De la tour d'ivoire à la place publique. Politique, 1(1), 9-18. https://doi.org/10.7202/040389ar

Ce document est protégé par la loi sur le droit d'auteur. L'utilisation des services d'Érudit (y compris la reproduction) est assujettie à sa politique d'utilisation que vous pouvez consulter en ligne.

https://apropos.erudit.org/fr/usagers/politique-dutilisation/
Cet article est diffusé et préservé par Érudit.

Érudit est un consortium interuniversitaire sans but lucratif composé de l’Université de Montréal, l’Université Laval et l’Université du Québec à Montréal. Il a pour mission la promotion et la valorisation de la recherche. https://www.erudit.org/fr/ 


\title{
De la tour d'ivoire à la place publique*
}

\author{
Gérard Bergeron \\ École nationale d'administration publique
}

«Les intellectuels et le pouvoir»: comment, exerçant ce métier de politicologue, n'être pas intéressé à traiter d'un pareil sujet surtout en une circonstance comme celle qui nous réunit? C'est peut-être le titre de discussion le plus... (disons) «croustillant» pour rassembler des gens de notre espèce.

Je proposerais de lui apporter une correction mineure. Heureusement, le titre dit «Les intellectuels...» et non pas «L'intellectuel...», qui est une abstraction sans être une essence: il n'y a que des intellectuels. Mais le titre poursuit: «... et le pouvoir» (sous-entendu: politique). Il serait préférable de parler des pouvoirs, non seulement parce que, affligés de la forme fédérative de gouvernement, nous en avons au moins deux sans prendre en compte les paliers municipal et international. Il y a en outre les pouvoirs économiques, culturels et scientifiques (où se situent les universités et les organismes de recherche), informationnels (communications et média). La forme plurielle s'impose autant pour les pouvoirs que pour les intellectuels. Mais il y a aussi un troisième terme, le plus indispensable, ô combien! qui sont les «clients» des intellectuels. Ils constituent la catégorie très large des citoyens autres que les intellectuels, qui sont situés en dehors des pouvoirs ou qui, simplement, n'ont pas de pouvoir.

* Texte présenté au colloque de l'ACFAS à Sherbrooke en mai 1981. 
Ce troisième terme est nécessaire, qu'il faut mentionner explicitement ne serait-ce que parce que les intellectuels, à part une poignée d'experts et de consultants, sont rarement en interface avec les titulaires ou praticiens effectifs des pouvoirs (sauf, peut-être et très partiellement, dans l'exercice de notre métier avec le pouvoir universitaire, ce qui n'est pas le sujet). C'est donc via la «clientèle» virtuelle des intellectuels que ceux-ci entrent en rapport indirect avec les pouvoirs. Première constatation qui fait voir l'inégalité et la dissymétrie entre cette catégorie des intellectuels, de toute façon restreinte, avec la clientèle universelle des pouvoirs politiques qui rejoignent, par définition, l'ensemble de tous les citoyens. La forme d'influence, que sont susceptibles d'exercer les intellectuels en rapport aux pouvoirs, est donc médiate, partielle, transitoire même et, conviendrait-il d'ajouter, tout ce qu'il y a d'aléatoire.

J'ajoute une dernière précision. Quand les intellectuels s'interrogent sur leur rôle et leur influence réelle, présumée ou désirée, ils nous apprennent moins sur les pouvoirs que sur eux-mêmes: sur Sartre, Mauriac, Aron, Galbraith, Soljenitsyne, Chomsky ou Althusser. (Je prends mes exemples ailleurs pour une gamme plus variée...) Ce sujet des intellectuels et des pouvoirs, il n'y a guère que les intellectuels à le soulever et il les incite aisément à l'auto-justification. Le traitement qu'ils en font comporte souvent d'assez claires tonalités auto-biographiques. Je tiendrais toutefois à répéter ce que j'ai déjà dit dans des contextes analogues: dans ce que je vais dire il n'y a aucune intention de prescrire ou de prohiber quoi que ce soit. Chaque membre de la confrérie intellectuelle a à établir sa propre règle de cohérence et de responsabilité: je ne vois pas d'autre principe défendable absolument.

Les intellectuels sont la première conscience critique de la société: ce peut être posé en proposition générale, qui résume 
tout le sujet de leur rapport aux pouvoirs. Mais ce serait à condition de donner aux mots «conscience» et «critique» leur maximum de sens: le premier terme comprend «science» et le préfixe attire l'attention sur la communauté, «science avec "; ainsi, "critique» doit-il s'entendre dans tous les sens, moral, psychologique et scientifique.

Qui sont les intellectuels? Je n'entrerai pas dans le dédale des catégorisations possibles. Court-circuitons en posant que ce sont d'abord ceux qui se considèrent et qu'on considère tels, ce qui fait d'ailleurs un bon nombre de paroissiens! Cette notion ne s'occupe pas des degrés d'éducation, et de gradation de diplômes, d'activités des cols blancs ou bleus et toute autre espèce de clivages d'origine, de profession, de revenu ou de statut social. Être un intellecutel, c'est surtout avoir tel tempéramment et telle personnalité et non pas seulement avoir une telle formation ou exercer telle activité. Ainsi donc, on peut être dans une carrière dite statistiquement «intellectuelle» et n'en être pas un - et vice-versa. Ovide Plouffe est un intellectuel; ses frères Napoléon et Guillaume ne le sont pas. On comprendra que je ne multiplie pas les exemples de la vie culturelle locale.

Un intellectuel, c'est quelqu'un qui organise sa vie en rapport aux choses de l'esprit, à la vie des idées - ce qui ne le rend pas plus intelligent ni intelligible. Plus que la moyenne des gens, il consent à l'activité de l'intellect ce qui risque de le mener à l'intellectualisme. Les idées sont son lot quotidien, il en produit parfois, il en consomme beaucoup, il en discute tout le temps; il aime surtout en manipuler (je n'ai pas dit: en jongler). C'est un aimable Pierrot Lunaire qui ne s'est jamais complètement débarrassé de la merveilleuse illusion que «les idées mènent le monde» - ou, tout au moins, le devraient. Cette même illusion mène à croire que plus son esprit s'applique à la considération des pouvoirs, plus on les connaît et que plus on connaît les choses du pouvoir, plus il devient facile de se 
définir par rapport à elles. On sait bien que la chaîne de gradation causale va plutôt dans l'autre sens...

Auriez-vous déjà pensé qu’à part la catégorie assez floue des «artistes» le seul groupement professionnel qui soit d'emblée rangé en totalité sous la notion d'intellectuels est celle des professeurs d'université? Et pour cause: fait-on plus intellectuel que de chercher à dominer un savoir pour ensuite tenter de le communiquer à d'autres? Ce serait notre titre de gloire pour ceux que cela impressionne, mais aussi notre tare pour ceux qui, nous le leur rendons bien, ont leurs raisons de se méfier de nous.

Les universitaires qui œuvrent dans le domaine des sciences dites humaines ou sociales ne sont jamais bien loin, de par l'objet de leurs travaux, de l'un ou l'autre aspect de l'exercice des pouvoirs. Mais c'est en science politique que le rapport est le plus direct: nous nous occupons des pouvoirs; nous ne faisons que cela sous l'un ou l'autre découpage de nos spécialités respectives; nous sommes même les seuls professionnels, avec les journalistes politiques, à interpeller, d'une façon ou l'autre plus ou moins médiée, les pouvoirs. Parmi les intellectuels, nous sommes les «spécialistes» des pouvoirs, ce qui supposerait que les autres intellectuels seraient des espèces d' «amateurs». Comme professeurs d'université et de collège, nous sommes des gens dont les services ont déjà été légitimés par la société, qui sont encore payés et entretenus par l'État pour faire la critique du rôle qu'il joue comme encadrement protecteur de la vie sociale. Faut-il que notre rôle soit jugé indispensable, ou que notre influence réelle lui soit bien inoffensive, pour que lui, l'État, permette une telle libéralité! 
À ce point de ma rédaction, je jette un coup d'œil au programme et constate que les ateliers précédents ont déjà traité de liberté et d'éthique, de consultation et d'entrepreneurship, ce qui m'incite à ne pas quitter le niveau de plus grande généralité qui est celui du sujet de ce matin. Mais non sans avoir remarqué au passage qu'au Québec, j’allais dire dans le grand village du Québec, les intellectuels et singulièrement les politologues occupent une certaine position privilégiée, qu'envient, en l'exagérant du reste, nos collègues du Canada anglais et d'autres pays. Le réseau est, en effet, court qui part du Devoir s'embranche aux Affaires publiques de Radio-Canada, que double maintenant Radio-Québec et que triple, en suppléance pour cause de grève, TVA, circuit qui se connecte encore dans les pages de «dossiers», de journaux et dans les entreprises de sondages pour s'épanouir, finalement, en multiples commandites et subventions - de qui vous pensez? - des pouvoirs, bien sûr! Mais comme ce dernier point n'est pas le propos du jour, je ne fais que l'esquisser en passant.

Retour au propos principal, les deux expressions célèbres de Benda sur «la trahison des clercs» et de Gramsci sur «l'intellectuel organique» signaleraient assez bien les deux cornes du débat. La formule de Benda est facilement tenue pour suspecte du fait de la position de celui qui l'évoque dans tel contexte donné. Quant à la notion de Gramsci elle peut aussi s'entendre d'une façon inversée, qu'on pourrait caractériser, selon une expression bien de chez nous, comme une situation où l'intellectuel organique risque, même si c'est en douce, de «se faire organiser». Il ne s'agit pas tant de la menace de corruption ou d'intimidation, cas trop clairs pour valoir un déve- 
loppement, mais d'une situation de services attendus où l'intérêt, et donc aussi l'ambiguïté, peut aller en l'un et l'autre sens. Ainsi, les pouvoirs semblent leur faire beaucoup d'honneur aux intellectuels dans l'acte, apparemment humble, de sembler les "écouter", voire même de les prendre comme inspirateurs. Dans la réalité de ce type d'échanges, les pouvoirs, sans perversité particulière, sont amenés à utiliser les intellectuels en des symbioses inégales qui sont souvent au profit des plus forts. Le propre d'un pouvoir c'est d'être intéressé, vérité qui est encore plus certaine que le désintéressement présumé de l'intellectuel.

Le pouvoir fascine. Comme l'argent qui en est une forme. Les intellectuels ne sont pas immunisés de ces fascinations, qui ne sont pas que celles qu'exercent les grands pouvoirs, politiques ou économiques. Une des premières leçons de science (naturelle) politique que $j$ 'aie eue en tout début de carrière fut lorsqu'à l'occasion d'une nouvelle nomination de doyen et de la création d'un nouveau département dans ma faculté, une série de postes universitaires devinrent vacants. Ils ne le furent pas longtemps: en une courte bousculade ils furent tous occupés. Bien entendu, ce type d'affectation se passe sous le couvert ennoblissant du sacrifice personnel, de la solidarité institutionnelle, du devoir d'être présent à cet endroit et à ce moment-là, car autrement ça serait pire! J'ai compris alors de façon définitive que les universitaires sont du monde comme tout le monde. Que le pouvoir fascine, la preuve en est que nous ne manquons jamais de candidats pour n'importe quel poste à n'importe quel niveau de prestige ou d'influence réelle.

Les pouvoirs fascinent les intellectuels, et sans doute pas moins parmi eux ceux qui se sont fait un métier de les étudier. Aussi les intellectuels peuvent-ils devenir enclins à s'en constituer un motu proprio, à titre personnel ou individualiste mais pour des raisons déontologiques ou civiques. Chercher, 
écrire, enseigner, vouloir atteindre un plus vaste public: c'est une chaine naturelle. Mais, sous le coup d'une profonde conviction, la communication des messages peut prendre des accents plus pressants. Dans Big City Politics (1965), E.C. Banfield rapporte: "In politics, a politician said, there are two kinds of people you have to watch out for professors and military men. They seem to think that they can go around giving order to people. " Entre nous, on sera d'accord pour maintenir la distinction: s'il se trouve des intellectuels à ce point engagés, qu'ils semblent donner des ordres plutôt que des opinions, les militaires n'ont pas d'abord à fonctionner à la persuasion intellectuelle. La boutade du politicien américain rapportée par Banfield fait penser au mot de Voltaire (cité de mémoire): "Je ne pense pas comme vous; mais je me battrai à mort pour que vous ayez le droit de vous opposer à ce que je pense».

L'expression topologique de la tension intérieure de l'intellectuel dans son rapport aux pouvoirs, je la figurerais par les deux positions de la tour d'ivoire et de la place publique. Pour en arriver à maîtriser le petit secteur de connaissance de sa spécialité et, si possible, le faire avancer quelque peu, s'impose l'exigence d'austérité de la tour d'ivoire qui n'assure pas le confort qu'on présume souvent. Mais si l'objet de cette recherche solitaire porte sur la chose publique, le retranché de la tour d'ivoire ne peut vraiment jamais oublier sa condition de personne civique. Cette conscience peut l'habiter de façon plus aiguë que tout autre praticien des sciences sociales. Et la place publique offre ses attraits, présente ses tentations à visage de facilité. Pourquoi, en effet, ne pas faire acte de présence sur la place publique avec le petit bagage amassé dans la tour d'ivoire? 
Une pareille tension peut aller jusqu'à aboutir à une espèce de déchirement intérieur. Théoriquement, il n'y a pas de problème: le principe de cohérence est dans la personnalité globale, où peuvent coexister la personne civique et la personne scientifique et même toutes deux s'entraidant. De fait, la tour d'ivoire, trop loin, la place publique, trop près, ont des exigences simultanées qui ne sont pas toujours aisément conciliables, quoi qu'on dise. L'option définitive pour l'une peut régler la question. Privilégier par alternance, ou par simple intermittence, l'une ou l'autre est d'un arrangement convenable si l'on peut s'accommoder d'une certaine mauvaise conscience de la tour d'ivoire quand on est sur la place publique, et vice versa quand on est dans la tour d'ivoire. Le difficile c'est d'avoir tout le temps un pied dans l'un et l'autre lieu et de penser qu'on peut ainsi y fonctionner avec un maximum d'efficacité. C'est trop pour un seul homme...

Ces propos nous ont mené progressivement à la notion d' «engagement», de laquelle nous aurions pu partir, ou à celle, plus récente et moins contraignante, d' «implication». Comme il n'y a pas telle chose qu'un «parti intellectuel» si ce n'est en une certaine littérature polémique, ni encore moins un parti des intellectuels, ce qui serait une horreur, cette question nous amène à la considération des partis politiques ou des partis tout court. Pour d'aucuns l'implication ou l'engagement doit épouser les formes et les exigences de la solidarité partisane. Pour d'autres, afin d'avoir la profondeur et la liberté requises, implication et engagement civiques de l'intellectuel gagnent à être extra-partisans. C'est un point de vue plus que défendable pour ménager sa liberté entière au sein d'un engagement civique profond. Mais c'est aussi gênant de participer à la conscience critique d'une cause, en dehors de ceux qui, aux premières lignes de combat, en font la promotion concrète. Voir l'histoire de tous les partis de gauche qui sont ceux qui 
attirent surtout les intellectuels, où ils s'y trouvent presque toujours et presque tous en «ménage difficile..."

Le peu de temps qui reste employons-le à soulever des questions que je qualifierais d'auto-protection prudentielle des intellectuels.

D'abord, ne pas s'attendre à, ni réclamer un régime d'immunité lorsque nous descendons sur la place publique. Si nous avons ce qu'une formule un peu pompeuse appelle un «magistère d'influence» c'est tant mieux, ou tant pis, mais nous n'avons aucun droit à revendiquer quelque statut privilégié, différent de celui de toute autre personne civique dans un pays libre.

Ensuite, nous avons à résister à la tentation d'entrer dans le jeu des actions par personnages politiques interposés. Si nous voulons «faire de la politique» faisons-en carrément, publiquement et à visière levée, aux risques et périls du genre de l'action partisane. Cela peut ne pas donner grand chose: on se sera au moins contenté. Mais cela peut aussi mener jusqu'à des leaderships de parti et à des postes de premier ministre, comme notre milieu en fournit trois exemples célèbres.

Enfin, si nous voulons continuer d'agir en plénitude de cette «conscience critique de notre société» dont je parlais au début, il importe de nous maintenir le plus loin possible des pouvoirs et de leurs détenteurs. Pas tellement pour des raisons de pureté et d'intégrité mais pour des raisons de la nécessaire distanciation pour une objectivation maxima. Un homme de ma génération qui a fait une carrière en science politique s'est trouvé à être à «Tu» et à «Toi » avec quelques-uns des plus importants ministres des gouvernements de la Révolution 
tranquille, du French power et du Bon gouvernement du Parti québécois. Les reliquats, parfois rancuniers, d'amitié ou de camaraderie de naguère ne doivent pas interférer avec les jugements critiques de l'heure. Faire abstraction de tels sentiments peut être d'un certain dommage au plan psychique, car, de bien entendu, il n'y a pas de valeurs humaines essentielles que des politiques. Ces aveux, on pourrait se les dire à l'âge de la rédaction de ses mémoires, si quelques-uns d'entre nous en avaient la faiblesse.

Finalement, je me demande si je ne suis pas en train de plaider pour une certaine gratuité dans la relation que nous nouons avec les pouvoirs. "Gratuité » je l'entends sur les résultats, la rentabilité de notre action de pensée, non pas sur ses fondements civiques qui restent toujours d'une belle et même terrible obligation. Pensons seulement à tout ce que nous avons reçu de cette société pour pouvoir y mener, en toute sécurité, une pareille activité. J'évoquais plus haut son rendement très aléatoire, un peu comme celui de bouteilles qu'on lance à la mer... Parfois, les messages qu'elles contiennent sont lus.

D'un mot, selon la dichotomie célèbre, comprendre ou changer le monde? Réponse: les deux. Pour changer le monde, il faut commencer par le comprendre; et, dans la mesure où on le comprend, comment ne pas vouloir le changer? Reste ouverte la question des moyens: les nôtres que nous impose la carrière sont limités mais, pour continuer à travailler, il faut garder la conviction qu'ils n'ont pas une portée triviale. 